\title{
Consideraciones en torno a "La fatal arrogancia"
}

La fatal arrogancia. Los errores del Socialismo es la última obra escrita por el recientemente fallecido Friedrick A. Hayek. (HAYEK, FRIEDRICH A.: La fatal arrogancia. Los errores del socialismo, Unión Editorial, Madrid, 1990. Tit. original: The fatal Conceit. The Errors of Socialism, Routledge y Chicago University Press, 1988).

\section{Introducción: ¿No habrá sido el socialismo un error?}

«El argumento fundamental de este libro -son las primeras palabras de Hayek- es que nuestra civilización depende (...) de (...) "un amplio orden de cooperación humana", más conocido por (...) "capitalismo"" (p. 33). Ese orden, no es -dice- fruto de intención, sino que deriva de ciertos procesos espontáneos (p. 33).

Nada más empezar, el A. presenta sus reglas de juego: el mejor de los posibles sistemas es el capitalismo; además, y es un segundo axioma, es necesario rechazar cualquier designio o intención.

Hayek comienza el desarrollo de estos dos postulados. El primer encausado es la moral. Sus normas (las de la moral) no proceden, para el economista austriaco, sino de una práctica que «fue generalizándose a través de procesos evolutivos basados en la selección» (p. 33). En efecto, el hombre tuvo que irse sometiendo a esas normas -tantas veces opuestas a sus deseos-, y esa sumisión le permitió alcanzar «la necesaria cohesión gracias a la cual accedieron sus miembros a un superior nivel de bienestar y conocimientos de diversa especie, lo que les permitió "multiplicarse, poblar y henchir la tierra" (Génesis, I, 28)») (p. 33). 


\section{TOPICOS}

Los socialistas -continúa- «perciben la realidad de manera distinta» (p.34). Y cabe asegurar que también lo hace cualquiera que ponga en entredicho los dogmas hayekianos... Pero esto, para el A., no tiene relevancia. El "enemigo" son los socialistas, y por eso presupone que cualquier no-socialista es un ferviente partidario de sus principios.

Para el A., los errores del socialismo son, fundamentalmente, dos. En primer término: «no existe ningún método conocido que permita a los diferentes actores descubrir cómo pueden orientar mejor sus esfuerzos al objeto de obtener el mayor producto posible para la comunidad» (p. 34). En efecto, para él, sólo el azar puede explicar la evolución de la sociedad.

En segundo lugar, Hayek considera que «la aceptación de las normas morales transmitidas por tradición (...) es lo que nos permite generar y utilizar un volumen de información y recursos mayor del que pudiera poner al alcance de la comunidad una economía centralmente planificada» (p. 34).

Hayek anatematiza: si la sociedad asume las recomendaciones socialistas en materia ética desaparecerá gran parte de la población y el resto quedará en situación de patente pobreza (cfr. p. 35).

Empeñado en la defensa de un deus ex machina -al que llamará también, con Adam Smith, "mano invisible" (p. 45)-, el A. austriaco manifiesta que es lógico que alguien se sorprenda de este descubrimiento, porque es genial (cfr. p. 36). ¡Hayek cree en un "misterioso"' proceso no racional, que conduce a un orden no intencionado!

\section{Captulo I: Entre el instinto y la razón}

«Para la mente primitiva debió resultar de todo punto inconcebible la existencia de un orden de actividades humanas de extensión 
superior a lo abarcable por la directa percepción de alguna mente ordenadora. Incluso para Aristóteles, que sólo mucha más tarde aparece en escena, un orden semejante nunca podria superar el perímetro alcanzable por la voz del heraldo (Ética, IX, X), por lo que entendía que ni siquiera cabía imaginar la existencia de un Estado de más de cien mil individuos (...). Aristóteles no aborda los problemas que nos ocupan sobre la base de la reflexión y la observación, sino sobre la de sus instintos» (p. 41).

La frase inicial del primer capítulo de La fatal arrogancia muestra varios aspectos interesantes del pensamiento de Hayek. Antes de nada, el uso de la conjetura: debió resultar... Por una parte, da la impresión de que desea dejar abierta una puerta trasera para una eventual "retirada intelectual"; de otra, una vez realizada la afirmación, la aplica de manera casi dogmática al resto de la argumentación.

Hayek intenta convencer al lector de una tesis que, según él, arranca de tiempos ancestrales. Sin embargo, no dedica ni una palabra a la cuestión del origen del hombre.

Las normas que Hayek va definiendo axiomáticamente constituyen, según él, uuna nueva y diferente moral (...) encaminada a reprimir la "moral natural", es decir, ese conjunto de instintos capaces de aglutinar a los seres humanos en agrupaciones reducidas, asegurando en ellas la cooperación, si bien a costa de entorpecer o bloquear su expansión" (p. 43). Surge, entre otras muchas, la siguiente cuestión: ¿qué extraño concepto tiene Hayek de "moral natural"? Además, presenta la cooperación (luego la denominará también solidaridad), como uno de los mayores obstáculos del capitalismo. No es la libertad -podemos añadir, sacando conclusiones que él no formula, pero que están implícitas- la que mueve la economía de mercado, sino el mero egoísmo. 


\section{TOPICOS}

Asegura Hayek que «la supresión de las tendencias instintivas (son) el mejor modo de coordinar las actividades de un gran número de personas» ( $p .43$ ). Pero, por qué motivo, o quién lleva a superar esas tendencias. La respuesta es elusiva: se limita a afirmar que no habriamos llegado donde lo hemos hecho si no hubiese sido «ignorada la recomendación de que todo semejante sea tratado con el mismo espíritu de solidaridad que se dedica a quienes habitan en el entorno más próximo» (p. 44). Pero, es lícito preguntar, ¿de dónde procede esa "recomendación"? Sobre este punto, Hayek calla. Pero no sobre la importancia fundamental del egoísmo: no estariamos donde estamos, repetirá múltiples ocasiones, si los hombres fueran solidarios...

Para argumentar esta tesis anti-solidaria, acude al "ejemplo límite": un mundo en el que todos tratasen a sus semejantes como a si mismos desembocaria en un mundo en el que pocos podrian fructificar. Si, por ejemplo, decidiéramos seguir los consejos que a favor de un comportamiento caritativo nos sugieren algunos medios de comunicación, someteriamos a los demás a graves carencias al distraer nuestro esfuerzo de las actividades que con eficacia sabemos practicar $y$ nos transformariamos en instrumentos de intereses sectarios sobre la importancia relativa de determinadas necesidades. En nada contribuiria, por lo demás, esa actitud a remediar los problemas que a todos preocupan (cfr. p. 44).

Pocas páginas después, encontramos una consecuencia lógica de los principios de Hayek. Afirma, con cierta carga de historicismo: «si la civilización es fruto de inesperados y graduales cambios en los esquemas morales, por mucho que nos desagrade, nos veremos obligados a concluir que no está al alcance del hombre establecer ningún sistema ético que pueda gozar de validez universal)) (p. 54). Sólo cabe la moral de "situación capitalista".

Una de las grandes ventajas de Hayek es que no exige ser leido entre líneas: dice todo lo que desea. Así: «admiro sin reservas a 
Darwin» (p. 58). Sin mediar más explicaciones lanza venablos contra quienes se han negado a aceptar las tesis evolucionistas. Asegura que uno de los grandes obstáculos de la teoria de ese pensador-que, por lo demás, considera "que es aplicable a cualquier campo»- fue la superstición de considerar que dondequiera que se advierta la existencia de orden debe suponerse la presencia de un ente ordenador (cfr. p. 59).

Una vez "dogmatizada" la evolución, comienza a sacar consecuencias: «los procesos de transferencia -afirma- por vía del aprendizaje de los usos culturales tiene lugar con una rapidez incomparablemente superior de lo que acontece en la evolución biológica» (p. 60).

Al igual que la evolución biológica, la evolución cultural está sometida al azar: todo proceso evolutivo -sea cultural o biológicoimplica la incesante adaptación a un conjunto de imprevistos acontecimientos cuya variación nadie puede prever (cfr. p. 61).

Satisfecho está Hayek del darwinismo, ya que le permite explicar "coherentemente" su teoria de la "evolución cultural hacia el liberalismo"; llega a afirmar que el darwinismo constituye uno de los más notables logros de la humanidad, ya que, gracias a él, se ha conseguido alcanzar una nueva visión del cosmos (cfr. p. 62).

Resulta muy interesante considerar que Hayek, el gran detractor del socialismo, coincida sobre este punto con sus mortales enemigos. Así escribía Marx a Engels, en diciembre de 1860: "He leido toda clase de cosas. Entre otras, el libro de Darwin sobre la selección natural. A pesar de toda la pesadez inglesa, este libro encierra del fundamento biológico de nuestra teoría" (K. Marx, Carta a Engels, diciembre de 1860. MARX-ENGELS: Gesammtausgabe, t. III, 2, p. 532). Y, poco tiempo más tarde: "La obra de Darwin parece muy importante y la acepto como base biológica de la lucha de clases en la Historia" (K. 


\section{TOPICOS}

Marx, Carta a Lassalle, 1861, Correspondencia Marx-Lassalle, en G. MAYER, p. 346).

El por qué de estas, en apariencia, extrañas coincidencias puede entenderse fácilmente. Una vez que unos y otros -marxistas y liberales- han perdido la posibilidad de encontrar una explicación para el mundo basándose en su Creador, se ven impulsados a encontrar alguna teoría que justifique la realidad. La que les resulta más asequible es precisamente el evolucionismo, ya que ahora pueden dar visos de credibilidad a una explicación sin necesidad de Dios. Cuando pensadores de nuestros días se refieren a la identidad de raíces de esos dos sistemas económicos, no atienden a los matices que los diferencian, porque descubren y ponen de manifiesto un planteamiento esencial: el desconocimiento del verdadero sentido de la dignidad humana.

\section{Capitulo II: Los origenes de la libertad, la propiedad y la justicia}

El segundo capitulo comienza también con una hipótesis: «parece razonable (...) situar el punto de partida del proceso civilizador en las regiones costeras del Mediterráneon (p. 65).

La propiedad plural -nombre con el que A. denomina la propiedad privada- surgió, según él, a medida que aumentaba el proceso de debilitamiento del espiritu de solidaridad (cfr. p. 67).

A continuación, Hayek hace un desarrollo de la historia de la humanidad: griegos, espartanos, romanos... Como en otras ocasiones, para dar relevancia a su tesis, elige enemigos. Por ejemplo: «hasta en Aristóteles y Platón rezuman nostálgicos anhelos de resucitar el modelo de la convivencia de la vieja Esparta (...). Se trata del retorno a ese tipo de micro-orden en el que el comportamiento de todos queda sometido a la exhaustiva supervisión de alguna omnisciente autoridad» (p. 69). 
Su animadversión contra lo que suene a socialismo, le lleva a simplificaciones no siempre basadas en hechos históricos ciertos: «la decadencia y colapso final de este primer orden histórico extenso sólo fue produciẻndose a medida que las decisiones de la administración central romana fueron desplazando a la libre iniciativa» (p. 69).

Los puntos más flojos de la tesis de Hayek se encuentran en cuestiones filosóficas. Su análisis del concepto de libertad es insuficiente, pues cae en la incompleta caracterización de la libertad como mero control del instinto ajeno: mi libertad llega, para él, hasta donde tropieza con la del otro.

Concluye este capítulo con un pequeño "ajuste" de sus tesis a la manera de espontaneidad: "el macro-orden espontáneo comprende, no sólo las decisiones económicas tomadas a nivel individual, "sino también" las que adopta cualquier organización que haya sido deliberada y voluntariamente establecida" (p. 76).

Captulo III: La evolución del mercado: el comercio y la civilización

Prosigue en este capitulo la explicación histórica: algunos tardaron en volver y temían no ser conocidos, y regresaban con ciertos presentes. De esa fase de regalos se llegó «a la aparición de instituciones de carácter más impersonal constituidas por intermediarios") (p. 84).

El hombre fue adaptándose a la nueva situación, en la que dejaba de ser un miembro de la tribu, para ser un ciudadano egoísta del mundo. Todo esto sin que los gobernantes tuvieran prácticamente ninguna influencia: «mucho se exagera en torno al papel desempeñado por los gobernantes en el devenir histórico debido a que, obviamente, nos ha llegado mucha más información acerca de lo que ellos hicieron que de lo que la coordinada actividad individual iba consiguiendon ( $p$. 87). 


\section{TOPICOS}

De nuevo Aristóteles recibe las iras del A. La afirmación aristotélica de que "un colectivo excesivamente numeroso no puede participar en el orden", merece para Hayek un absoluto desprecio.

Cierta explicación de la animadversión hayekiana contra Aristóteles la tenemos pocas páginas más adelante. Lo que está preparando en realidad es una descalificación de la Iglesia Católica, partiendo de que ésta ha asumido -según el A.- acriticamente los principios aristotélicos: «los efectos de la sistematización aristotélica de una ética correspondiente al micro-orden fueron reforzados por la influencia que dicho filósofo ejerció sobre Santo Tomás de Aquino en el siglo XIII, la cual dio lugar a que, más tarde, tal línea de pensamiento llegara a convertirse, de hecho, en la doctrina oficial de la Iglesia. La animadversión hacia la práctica del comercio, que ha prevalecido tanto en la Iglesia de entonces como en la de ahora, su condena de cobro de intereses -que antaño se equiparó con la usura-, su defensa del precio justo y su displicente tratamiento del beneficio, son ideas impregnadas de pensamiento aristotélico") (p. 91).

Las lagunas e imprecisiones de esta afirmación son numerosas. Señalaremos sólo una: la condena de la usura no fue causada por el interés, sino por el "exceso" de intereses solicitados, que empobrecian a los demás. Y porque, a diferencia de lo que ocurre muchas veces en nuestros dias, el dinero obtenido en préstamo no iba destinado a la consecución de beneficios sino a cubrir necesidades inmediatas.

Tras considerar con gran acierto, que la información es algo fundamental en el mundo económico, vuelve a reiterar su creencia en el azar. El avance, en efecto, se produce «sin que para ello tenga nadie que ser consciente de la mayor parte de los detalles del orden en que se desarrolla su propia actividad». Añade: «pese a todo ello, y como volviendo la espalda a esos grandes avances intelectuales, una visión animistica, ingenua y elemental de la realidad -una vez más 
ornada en ribetes aristotélicos- surgió entre los estudiosos de las ciencias sociales y ha servido de fundamento del ideario socialista" (p. 91).

Resulta, pues, que para Hayek socialismo y doctrina social se identifican en sus raices: la falta de rechazo del concepto de solidaridad y, en definitiva, la no aceptación de un deus ex machina o "mano oculta" que explique el avance del mundo. Unas, porque pretenden orientar el avance -los socialistas-; otros -la Iglesia-, porque creen en principios sobrenaturales (supersticiones, las denomina ${ }^{\circ}$ en ocasiones), las dos posturas están radicalmente equivocadas.

\section{Capitulo IV: La rebelión del instinto y la razón}

Hayek se dispone ahora a responder a sus objetores. El primero es el "constructivismo": "conduce forzosamente a una falsa interpretación de la naturaleza y la verdadera esencia de las instituciones que facilitan la pacífica convivencia» (p. 94). Aquí queda englobado Descartes, del que afirma que sus «pretensiones carecen de todo fundamento') (p. 94).

Otro encausado es Jean-Jacques Rousseau. Es acusado de ser cartesiano, y de más cosas: sus tesis uhicieron olvidar que, para conseguir el orden social, es preciso fijar limites al comportamiento individual» (p. 95). El principal error de Rousseau -para Hayek- es no haber dicho que es preciso dominar los instintos: «quizá constituya este último hecho la raiz más profunda de esa fatal arrogancia de que es victima del racionalismo moderno en virtud del cual se intenta restituir a la humanidad una paradisiaca situación que supuestamente le permitirá "dominar la tierra" -cual nos ordena el Génesis-, no a través de la sumisión a los aprendidos esquemas restrictivos del comportamiento, sino sobre la base de dar rienda suelta a nuestros más primitivos instintos») (p. 95). 


\section{TOPICOS}

Haciendo decir a Rousseau más de lo que dijo, Hayek asegura que fue el causante de todos los males morales de la moderna civilización.

Hayek concluye así su alegato anti-sociológico: la sociología prefiere ignorar los avances de esfuerzos investigadores dedicados al estudio de las complejas estructuras hoy surgidas en el derecho, el lenguaje y el mercado (cfr. p. 97.)

A punto de acabar el primer apartado del capitulo IV, Hayek escribe algo sorprendente, no sé si es más por la humildad con que comienza o por la arrogancia con la que concluye. "Tanto las limitaciones de espacio como mi propia falta de competencia al respecto me impiden abandonar en la segunda de las instituciones cuestionadas: la unidad familiar. Quisiera, sin embargo, dejar constancia de que, en mi opinión, los últimos avances cientificos ponen en tela de juicio muchas de las recomendaciones morales relativas a la sexualidad, siendo lo más probable que en torno a estas cuestiones se produzcan a no tardar importantes cambios»" (p. 98). Quien se proclama falto de competencia acaba siendo científicoprofeta de liberalizaciones sexuales.

La religión sufre nuevos embates. Esta vez, Hayek la convierte en mero instrumento de sus tesis. Se manifiesta de acuerdo con autores como Monod al rechazar el origen religioso de la moral. Añade: al rechazar esa interpretación religiosa, no conviene olvidar que, gracias a las religiones, han perdurado ciertos hábitos que han permitido a la humanidad alimentar un mayor número de bocas de lo que hubiera resultado posible a través de la estricta aplicación de los dictados de la razón (cfr. p. 105).

Criticadas las personas, pasa a diversas teorias: el racionalismo, el empirismo, el positivismo y el utilitarismo: (todas (...) coinciden en su rechazo de la moral tradicional" (p. 110). (No olvidemos que lo que

- Hayek denomina moral tradicional nada tiene que ver con la moral natural). 
Si todos no quedasen convencidos de la conveniencia de aceptar la moral por él propuesta, añade un anatema apocalíptico: «habrá sin duda quien se pregunte si tanto sacrificio vale la pena, convendrá en cualquier caso, recordar siempre que la única alternativa a la civilización eș la pobreza y el hambre» (p. 113).

Para quien tenga "escrúpulos" sobre esta "nueva moral tradicional" cuyo objetivo es el beneficio, Hayek ridiculiza esos «sentimientos de culpabilidad o de conciencia social» (p. 115).

\section{Capitulo V: La fatal arrogancia}

El A. afirma que, en su opinión, el socialismo desea repensar la moral tradicional, y el derecho y el lenguaje, para encontrarles fundamentos racionales. Pero esa carencia -que reconoce y defiendede fundamento racional, no le preocupa lo más mínimo. Es más, considera que incluso los posibles sistemas morales alternativos carecen también de justificación racional.

El A. se detiene en una justificación "racional", no de su "moral tradicional", sino de la evolución histórica por la que ésta ha ido cuajando. El ejemplo inicial lo repite con insistencia a lo largo de la obra: los principios morales que fundamentaban las sociedades primitivas -solidaridad, altruismo, decisión del grupo, etc.- "son incapaces de alimentar a la numerosa población que hoy puebla el orbe» (pp. 122.123).

Para Hayek, las circunstancias que rodean el actuar de cada hombre no pueden aceptar el encuadre en unos principios racionales: existe un cúmulo de decisiones causadas meramente "irracionales".

Tropezamos ahora con uno de los mejores resúmenes del pensamiento hayekiano: upese a ser indudable que el "bienestar material carece de principios" (...), la propensión a rechazar las 


\section{TOPICOS}

normas de convivencia que han hecho posible la existencia de tal tipo de orden (...) deriva del conocimiento de que el logro del bienestar económico debe regirse por algún principio, así como de la negativa de aceptar (...) que el orden social extenso implica la existencia de procesos de carácter competitivo en los que el éxito no se debe a una mente superior, a un comité, a algún lugar sobrenatural o a la conformidad con algún principio conocido de mérito individual» ( $p$. 127).

Ese proceso evolutivo irracional es lo que -según Hayek- ha permitido llegar a la civilización actual. Si alguna mente ordenadora -asegura el pensador austriaco- hubiera intervenido, habria procurado evitar las diferencias sociales o los dolores provocados por ese desarrollo. De ese modo, no hubièramos alcanzado el nivel del que ahora disfrutamos. Por eso, escribe, es preciso afirmar que «la evolución no puede ser justa) (p. 128). Si se hubiesen reprimido las diferencias, se habria detenido la posibilidad de nuevos avances.

Nuestros antepasados, ni siquiera sospecharon las inmensas ventajas que tendria vivir esas normas que ellos inconscientemente iban desarrollando, ya que «sólo sometiéndose a un conjunto de normas cuya obediencia a veces le resulta incómoda logrará un grupo competir con éxito con otros que antes descubrieron y asumieron esa adecuada normativa) (p. 131).

Hayek no sólo considera que el capitalismo sufriria si a alguien se le ocurriera ser altruista; resulta que «carece de sentido recomendar a cualquier sujeto que oriente su comportamiento sobre motivaciones altruistas" (p. 138).

Como sorprendido por sus propias afirmaciones, algo más abajo, Hayek matiza algo sus tesis. No se sabe a cuento de qué -desde el punto de vista de la coherencia intelectual-, recomienda que sobre la base de los propios ingresos, se dedique algo a la satisfacción de esa 
instintiva inclinación a atender las necesidades de nuestro próximo entorno.

\section{Capítulo V: El misterioso mundo del comercio y del dinero}

Al comenzar un negocio, se arriesga tiempo, esfuerzo, y principalmente, información. Este último factor es de radical importancia para llevar adelante cualquier empresa económica: si alguien fuese pregonando a los cuatro vientos los secretos de su éxito económico, es evidente que muchos otros intentarian entrar en ese mismo sector. Precisamente esa reserva de datos es lo que -según Hayek- ha llevado a muchos a sentir una gran hostilidad hacia el comerciante, ya que éste estuvo siempre envuelto en una aureola de misterio.

Esta situación, considera el A., se ha visto agudizada por la doctrina marxista, que ha considerado que la única verdadera fuente de riqueza es el trabajo material. Por fortuna, continúa Hayek, esa extraña visión va siendo superada paulatinamente.

Es cierto, considera, que el que un mero intercambio de bienes incremente las ganancias de cuantos en él intervienen fue y sigue siendo una conclusión dificil de captar intuitivamente. Muchos continúan negándose a valorar adecuadamente ese esfuerzo meramente mental que caracteriza a la actividad comercial (cfr. p. 155)

Es cierto -son palabras suyas-, que «la creación de riqueza no es un proceso meramente fisico ni reducible a una concatenación de relaciones efecto-causa. No depende de ningún conjunto de hechos materiales objetivos, sino del aprovechamiento de la separada y diferente información poseida por millones de actores; información que, a modo de precipitado, queda recogida en los precios que orientan las posteriores decisiones" (p. 163). Pero no lo es tanto que 


\section{TOPICOS}

ésa sea la única verdad ni que se produzca una «desdeñosa actitud de los intelectuales hacia estas materias» (p. 164).

Para Hayek, ante el dinero. el hombre adopta una postura ambivalente. De una parte, se le considera como un instrumento de opresión. De otra, se le aprecia como salvaguardia de la libertad (cfr. p. 166).

El mundo del dinero y del crédito es, sin duda, uno de los más complejos y sorprendentes. Entre otras cosas, porque la economía es más un arte que una ciencia. Al explicar éste y otros aspectos semejantes, Hayek demuestra su aguda visión cuando de cuestiones estrictamente económicas se trata.

Considera que muchos niegan a la empresa su derecho a los beneficios y uen la medida en que tales actitudes afectan al bienestar de terceros, sólo pueden ser consideradas egoistas, al imponer a otros un ascetismo que indefectiblemente los sumirá en la pobreza y en toda suerte de privaciones» (p. 171).

\section{Capitulo VI: Nuestro envenenado lenguaje}

Aunque, a decir verdad, la cuestión parece no cuadrar mucho con el tema de fondo del libro, el sobrino de Wittgenstein ha incluido en su obra unas reflexiones sobre aquello a lo que su pariente dedicó toda una vida de estudios filosóficos.

Comienza el capitulo -como otros- con una presunción: «el comercio, las migraciones y la expansión y amalgama de poblaciones no sólo abriria los ojos a la gente; la harán también más locuaz) (p. 173).

A partir de este momento comienza un análisis de la función del lenguaje en la vida del hombre. De una parte, considera la gran influencia que en la vida tiene el mismo lenguaje y, de otra, cómo «las 
expresiones que normalmente utilizamos están siempre condicionadas por nuestra propia concepción o interpretación de la realidad» ( $p$. 174).

Después de estos comienzos "inocuos", Hayek pasa al "ataque filosófico". Su interés por el lenguaje está en encontrar nuevos apoyos para sus principios. Un ejemplo: «el propio término "orden" constituye un buen ejemplo de vocablo que, antes de Darwin, hubiera implicado la existencia de un acto personal. A principios de siglo pasado, incluso un pensador tan agudo como Jeremy Bentham no dudó en afirmar que "el concepto de orden presupone un fin"” (p. 175). Y, por si fuera poco: "el estudio de cualquier problema de alguna manera relacionado con la acción humana se encontraba profundamente impregnado de "animismo", sólo en parte paliado por la influencia de Adam Smith y su postulado de la "mano invisible"" (p. 175).

El socialismo se convierte en pluma de Hayek en otra manifestación de este tipo de interpretación animista que históricamente adoptaron diversas religiones (cada una sobre la base de las respectivas "deidades") (cfr. p. 176).

Los culpables de todos estos dislates son, para el pensador austriaco, Platón y Aristóteles, y, lógicamente, las religiones globalmente consideradas. Para Hayek, Platón y Aristóteles no entendieron en absoluto la existencia de procesos evolutivos; por eso, «abordan los problemas relativos a la humana convivencia cual si se tratara de ordenar tan sólo un específico e invariable número de individuos cuyas condiciones personales pueden ser plenamente conocidas por quienes se responsabilizan de la buena marcha del orden; o de un esquema de convivencia que, cual han pregonado los diferentes credos religiosos -incluido el socialista-, fuera fruto de previa intervención por parte de alguna mente superior” (p. 178). Da la impresión -perdónese el ex abrupto- que la única "mente 


\section{TOPICOS}

superior" que Hayek admite, pues todo lo entiende y sobre todo dogmatiza, es él mismo.

El último párrafo vuelve a ser una admonición para quienes no acepten sus tesis: «la ética anticapitalista (...) no ceja en su empeño. Sigue impulsando sin desmayo a la gente a rechazar precisamente aquellas instituciones que garantizan incluso su propia supervivencia. En nombre de la libertad se conculca la propiedad plural, la inviolabilidad de los contratos, la competencia, la publicidad, el beneficio e incluso la moneda. Empecinados en el ilusorio convencimiento de que el hombre puede alcanzar a través de la razón cuanto demandan nuestros innatos instintos, quienes asi argumentan se han convertido de hecho en una peligrosa amenaza para la civilización» (p. 190).

\section{Capitulo VIII: El orden extenso y el aumento de población}

El interés de Hayek por la población es de carácter meramente económico. Es importante hacer esta precisión porque algunos autores partidarios del respeto de la moral sexual han echado las banderas del viento tras leer lo que Hayek dice al respecto, sin detenerse en el por qué lo dice.

Hayek condena a quienes no consiguen entender el estrecho vinculo que une el incremento de la población con la favorable evolución del mundo civilizado (cfr. p. 193). De nuevo, la gran bestia negra es el socialismo, encarnación de ese mal que -de instaurarse- acabaría no sólo con las poblaciones futuras sino también con la actual civilización.

El A. insiste en que la expansión demográfica no sólo no daña a la economía, sino que permite «iniciar procesos de ininterrumpida aceleración hasta constituirse en el factor que fundamentalmente condicione cualquier ulterior avance de la civilización, en sus aspectos materiales o espirituales»! (p. 194). 
El análisis sobre el carácter regional del problema demográfico resulta sugestivo: coningún derecho tenemos a evitar el aumento de la población en otros lugares del mundo», porque «si no se interfiere en su evolución, tales poblaciones crecerán sólo en la medida en que puedan alimentarse a sí mismas» (p. 97).

Hayek considera que no hay ningún riesgo de que, en un futuro razonable (cfr. p. 198), acabemos con los recursos naturales. Por eso, afirma, seria presuntuoso inducir a ciertas poblaciones a contener su expansión. De ahí, su condena sin paliativos al Club de Roma.

El canto de Hayek a la diversidad de los componentes de la raza humana y el modo como esta diversidad da lugar a una mayor riqueza son interesantes. Desafortunadamente, como en otras ocasiones, produce pena que el A. no sea capaz de ver más allá de la pura rentabilidad económica.

\section{Capitulo IX: La religión y los guardianes de la tradición}

Reitera Hayek, al comienzo de este capitulo, su tesis de los dos "modos de vida". De uno, el de aquellos que viven unos para otros, persiguiendo fines comunes. «Estas arcaicas actitudes y emociones -asegura- son hoy defendidas por ciertas tendencias racionalistas y por el empirismo, el hedonismo y el socialismo asociado a las mismas» (p. 211).

De otro lado, el capitalismo. Es decir, un sistema en el que no tenemos porqué -incluso hay que decir: no debemos- preocuparnos por los demás: (nya no servimos principalmente a nuestros inmediatos allegados ni perseguimos fines comunes» (p. 211).

Llegar a esta segunda etapa supuso -para Hayek- un esfuerzo notable, porque hubo que ir superando los instintos $y$, también, los ataques de la razón. Precisamente aquí, como para encontrar un 


\section{TOPICOS}

"eslabón perdido" en su teoría, Hayek acude a la religión. Sus palabras suenan así: «las costumbres y tradiciones, adaptaciones no racionales al medio, pueden más fácilmente guiar la selección cuando cuentan con el apoyo del totem y del tabú, o bien de creencias mágicas o religiosas nacidas de la tendencia a interpretar cualquier orden humano conocido en términos animisticos) (p. 212). Triste resulta, sin embargo, la comparación de la religión, los tabúes y la magia...

Hayek reinterpreta la moral en su lucha contra el socialismo: «la visión religiosa según la cual la moral está determinada por procesos que no resultan comprensibles es mucho más acertada (aunque no exactamente en el sentido pretendido) que la ilusión racionalista según la cual el hombre, sirviéndose de su inteligencia, inventó la moral que le permitió alcanzar unos resultados que jamás habria podido prever»" (p. 213). Y, como si hubiese leído el San Manuel, Bueno, Mártir, de Miguel de Unamuno, añade: podemos comprender mejor a los clérigos que escépticos respecto a la validez de algunas de sus doctrinas, persisten en enseñarles ante el temor de que el abandono de la fe conduzca a una degeneración de la conducta moral.

Dentro de su linea anti-racionalista, Hayek considera a la religión como "ffalsas razones", que influyeron sobre los hombres para que éstos realizaran lo que exigía el mantenimiento de una estructura capaz de alimentar a una población más numerosa” (p. 215). Y añade: (así como la creación del orden extenso no fue fruto de previa intencionalidad, así tampoco existe razón alguna para suponer que el apoyo derivado de la religión haya sido deliberadamente fomentado, o que haya existido a este respecto una especie de "conspiración"" (p. 215).

La fatal arrogancia concluye, en fin, con una nota personal. De una parte, afirma que considera que Dios no es sino couna personificación de esa tradición de la moral o de los valores que hizo que (...) un grupo pudiera sobrevivir» (p. 21.7). Por otra, asegura que 
-en definitiva- la fuente del orden de las religiones adscriben «a una divinidad concebida antropomórficamente (...) podemos ahora interpretarla como algo no al margen del mundo físico sino como una de sus características, demasiado compleja para que cualquiera de sus partes pueda ofrecer una "imagen" o "diseño" de la misma" ( $p$. 217).

La última frase de la obra tiene tintes apocalípticos. Asegura Hayek: de que se acepte mi tesis, «puede depender la supervivencia de nuestra civilización» (p. 217).

\section{A modo de conclusión}

A lo largo de las páginas precedentes, hemos hecho referencias a algunos de los errores y contradicciones que se suceden en La fatal arrogancia. Sin ánimo de ser exhaustivos, mencionamos algunos: desprecio sistemático de la razón humana y sustitución por una evolución social fruto del azar; menosprecio y descalificación de todos los pensadores a los que cita, a excepción de Darwin; continuas generalizaciones y uso de lugares comunes; falta de consistencia en las afirmaciones de carácter histórico; carencia de rigor en las consideraciones de carácter científico; aceptación acrítica de la hipótesis evolucionista; falta de fundamentación metafisica; etc.

En esta obra se detecta, en fin, junto a interesantes consideraciones de carácter estrictamente económico, desconocimiento filosófico e histórico y una desmedida arrogancia que le llevan a convertirse en un nuevo profeta, que condena a todos aquellos que no aceptan sus tesis, porque -según él- sólo "su" capitalismo puede permitir la no aniquilación de la actual civilización. 
Copyright of Tópicos. Revista de Filosofía is the property of Universidad Panamericana and its content may not be copied or emailed to multiple sites or posted to a listserv without the copyright holder's express written permission. However, users may print, download, or email articles for individual use. 